Research in Astronomy and Astrophysics manuscript no.

(LTEX: Sharina.tex; printed on August 6, 2020; 1:10)

\title{
Age and chemical composition of the globular cluster NGC 6652
}

\author{
Margarita Eugene Sharina ${ }^{1}$ and Vladislav Vladimirovich Shimansky ${ }^{2}$ \\ ${ }^{1}$ Special Astrophysical Observatory, Russian Academy of Sciences, Nizhnii Arkhyz, 369167, Russia; \\ sme@sao.ru \\ ${ }^{2}$ Kazan Federal University, 18 Kremlyovskaya street, Kazan, 420008, Russia \\ Received 2020 January 23; accepted 2020 March 10
}

\begin{abstract}
We present the results of determination of the age, helium mass fraction (Y), metallicity $([\mathrm{Fe} / \mathrm{H}])$, and abundances of the elements $\mathrm{C}, \mathrm{N}, \mathrm{O}, \mathrm{Na}, \mathrm{Mg}, \mathrm{Ca}, \mathrm{Ti}, \mathrm{C}$ and $\mathrm{Mn}$ for the Galactic globular cluster NGC 6652. We use its medium-resolution integrated-light spectrum from the library of Schiavon and our population synthesis method to fulfill this task. We select the evolutionary isochrone and stellar mass function for our analysis, which provide the best approximation to the shapes and intensities of the observed Balmer line profiles. The determined elemental abundances, age and metallicity are characteristic of stellar populations in the Galactic Bulge.
\end{abstract}

Key words: globular clusters: general - globular clusters: abundances - globular clusters: individual: NGC 6652

\section{INTRODUCTION}

NGC 6652 is an intermediate metallicity $([\mathrm{Fe} / \mathrm{H}]=-0.96$ dex and $[\mathrm{Fe} / \mathrm{H}]=-0.85$ dex in the Zinn \& West (1984) and Carretta \& Gratton (1997) scales, respectively) ${ }^{1}$, old Galactic globular cluster (GC, 11.7 Gyr, Chaboyer et al. 2000). It is at the heliocentric distance of $10 \mathrm{kpc}$ and is mildly obscured by Galactic dust $(\mathrm{E}(\mathrm{B}-\mathrm{V}) \sim 0.1 \mathrm{mag}$ ) (Bica et al. 2016 and references therein). It is thought to be associated with the bulge (Bica et al. 2016) or with the inner halo (Chaboyer et al. 2000).

We did not find elemental abundances obtained in high-resolution spectroscopic studies for stars in NGC 6652 in the literature. Conroy et al. (2018) estimated the relative age (1.01 \pm 0.01$),[\mathrm{Fe} / \mathrm{H}]=$ $-0.93 \pm 0.02$ dex and abundances of $\mathrm{C}, \mathrm{N}, \mathrm{Mg}, \mathrm{Si}, \mathrm{Ca}$ and Ti for NGC 6652 using the integrated-light (IL) spectrum from Schiavon et al. (2005) and their updated stellar population models. Schiavon et al. (2005) found $[\mathrm{Fe} / \mathrm{H}]=-1.1$ dex using this spectrum (their Table 1).

1 The iron content in solar units is $[\mathrm{Fe} / \mathrm{H}]=\log \left(\mathrm{N}_{\mathrm{Fe}} / \mathrm{N}_{\mathrm{H}}\right)-\log \left(\mathrm{N}_{\mathrm{Fe}} / \mathrm{N}_{\mathrm{H}}\right)_{\odot}$, where $\mathrm{N}_{\mathrm{Fe}} / \mathrm{N}_{\mathrm{H}}$ is the ratio of the abundances of iron and hydrogen in terms of numbers of atoms, or in terms of mass, which is related to the mass fraction of elements heavier than 
The elongated orbit with the apocenter radius of $8.1 \mathrm{kpc}$ and eccentricity of 0.58 (Balbinot \& Gieles 2018), proximity to the Galactic center and rather low metallicity make NGC 6652 an interesting object of study in the context of Galactic evolution.

In this work we make use the integrated spectrum of NGC 6652 from Schiavon et al. (2005) and our population synthesis method (Sharina \& Shimansky 2019, Sharina et al. 2018, Sharina et al. 2017 and references therein) to derive the helium mass fraction (Y), age and abundances of several chemical elements.

\section{OBSERVATIONAL DATA}

The spectrum of NGC 6652 was obtained by Schiavon et al. (2005) with the Ritchey-Chrétien spectrograph mounted on the $4 \mathrm{~m}$ Blanco telescope at the Cerro Tololo Inter-American Observatory (CTIO) with the $1.5^{\prime} \times 5.5^{\prime}$ long slit oriented in the East-West direction. The wavelength range is 3360-6430 $\AA$ and the spectral resolution is $3.1 \AA$ full width at half maximum $(\mathrm{FWHM})$ in the central $\sim 1000 \AA$, deteriorating to $3.6 \AA \mathrm{FWHM}$ at the blue and red ends of the spectrum. According to the adopted strategy of the observations, the spectrum was obtained by drifting the spectrograph slit across the core diameter of the cluster ( $\pm 9^{\prime \prime}$ from the center of NGC 6652). The background spectrum was obtained at the angular offset from the cluster center of $12^{\prime}$ to the West. The spectrograph slit was trailed within the angular distance of $5^{\prime}$ for the sky background exposure.

The quality of the IL spectrum of NGC 6652 from Schiavon et al. (2005) is very good (please, see the original paper for the complete description of the observations, instrumental setup and spectra reduction). Although the authors do not report the resulting signal-to-noise ratio $(\mathrm{S} / \mathrm{N})$, they compare spectra of two moderately metal-poor clusters with similar metallicity ([Fe/H] -1.1 dex), NGC 6723 and NGC 6652, and demonstrate in their Fig. 3, that the number of stars in the blue part of the horizontal branch (HB) may influence the intensities of absorption lines in the spectra. Balmer lines are weaker and metal lines are stronger in the spectrum of NGC 6652 with the redder HB. These definite conclusions would not be possible from the visual comparison of the spectra with poor $\mathrm{S} / \mathrm{N}$. As will be shown in the next section, our analysis confirms that the $\mathrm{S} / \mathrm{N}$ in the spectrum is high. The probability of a noticeable contribution of Galactic field stars in the spectrum is low, despite the fact that NGC 6652 is located in a crowded stellar region.

\section{DATA ANALYSIS AND RESULTS}

The spectrum of NGC 6652 was compared with the synthetic IL spectrum computed according to effective temperatures $T_{\text {eff }}$, surface gravities $\log (\mathrm{g})$ and metallicities $[\mathrm{Fe} / \mathrm{H}]$ set by theoretical evolutionary isochrones for stars in the cluster. In this work, we use the scaled-solar isochrones by Bertelli et al. 2008 (hereafter: B08) and by the TERAMO group (Pietrinferni et al. 2013 and references therein, hereafter: TERAMO isochrones).

Synthetic spectral calculation is based on the plane-parallel, hydrostatic stellar atmosphere models by Castelli \& Kurucz $(2003)^{2}$. The calculated synthetic spectra of individual stars were summed according to the selected mass function (MF). In this work, we apply the MFs by Chabrier (2005) (equation (2) in 
that paper, hereafter: Ch05) and Salpeter (1955) (please see the paper Ch05 for a discussion about the relation between the initial and present-day MFs). Let us note that the preferred isochrone and the MF can

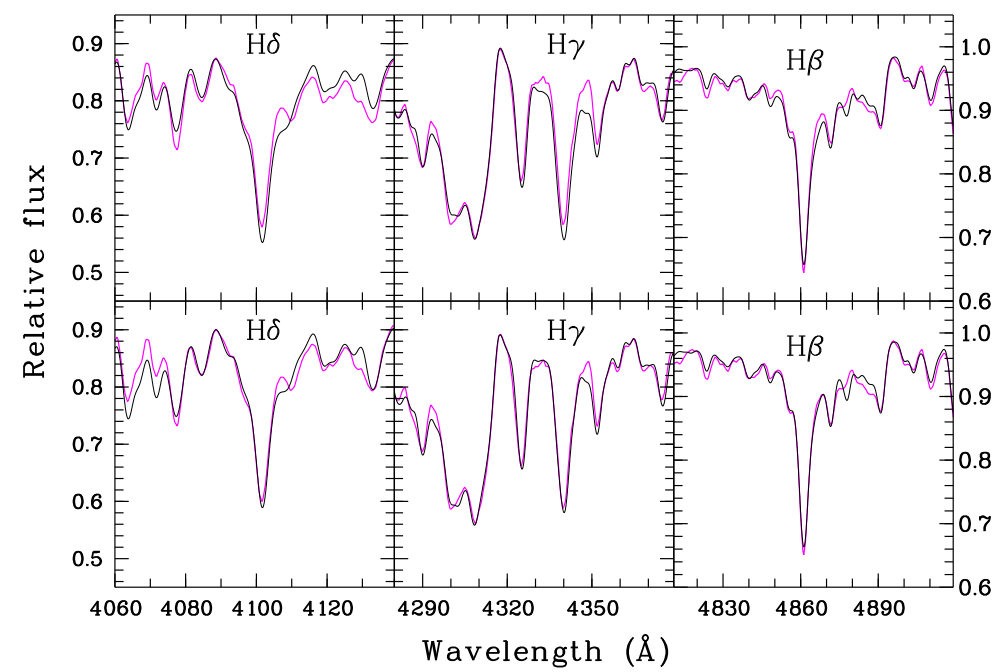

Fig. 1: Comparison of three Balmer lines in the continuum normalized spectrum of NGC 6652 (Schiavon et al. 2005) (magenta) and the synthetic IL spectra (black) computed using the MF by Ch05, the same elemental abundances but different isochrones: $\mathrm{Z}=0.002, \mathrm{Y}=0.26, \log (\mathrm{age})=10.15 \mathrm{~B} 08$ (bottom) and $\mathrm{Z}=0.002, \mathrm{Y}=0.248$, age=14 Gyr (TERAMO) (top).

be selected purely on the basis of comparing the strengths and shapes of Balmer lines in the observed and synthetic spectra.

The best correspondence between observational and theoretical data is illustrated in the bottom panels of Fig. 1. It can be seen that the synthetic spectrum computed with the isochrone $\mathrm{Z}=0.002, \mathrm{Y}=0.26$, $\log ($ age $)=10.15$ (B08) and the Ch05 MF better describes the observed Balmer lines than the spectrum computed with the TERAMO isochrone $\mathrm{Z}=0.002, \mathrm{Y}=0.248$, age $=14 \mathrm{Gyr}$.

This result can be understood if we consider the color-magnitude diagram (CMD) of NGC 6652. It can be ascertained in the left panel of Fig. 2 that the distribution of stars on the cluster CMD (Sarajedini et al. 2007) is described well by the isochrone $\mathrm{Z}=0.002, \mathrm{Y}=0.26, \log (\mathrm{age})=10.15$ (B08) for all evolutionary stages, except for the HB which is red in the case of NGC 6652. However, since there are hot luminous stars on the continuation of the cluster HB and of the Main sequence, and some of these stars may contribute to the integrated spectrum, one may conclude that this isochrone can describe the spectrum reasonably well. Fig. A.1 in the Appendix illustrates a comparison between the original non-smoothed theoretical IL spectra computed using different isochrones. Fig. A.2 displays the comparison between the observed continuum normalized spectrum of NGC6652 and the synthetic IL spectra computed using the same isochrone and elemental abundances but different MFs. It can be seen that there are no significant differences between the synthetic IL spectra computed applying the Ch05 and Salpeter (1955) MFs.

Figures A.4 and A.5 in the Appendix demonstrate how the changes in age and Y values influence the synthetic profiles of three Balmer lines in comparison with the continuum normalized IL spectrum of NGC 6652. We considered the following isochrones by B08 for this demonstration: (1) $\mathrm{Z}=0.002$, 

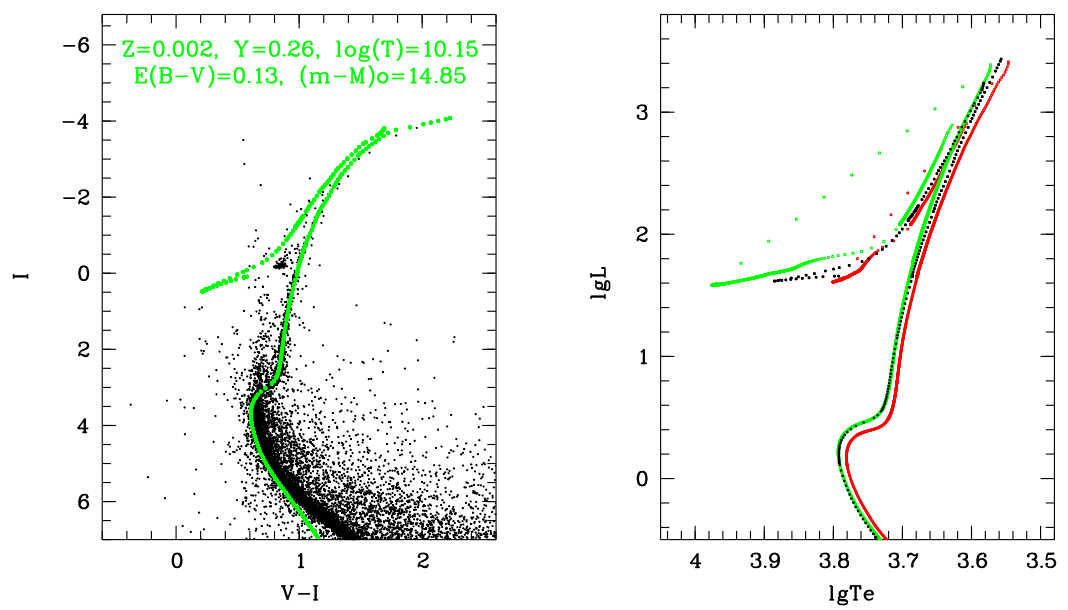

Fig. 2: Left: comparison between the CMD of NGC6652 (Sarajedini et al. 2007) and the theoretical evolutionary isochrone $\mathrm{Z}=0.002, \mathrm{Y}=0.26, \log (\mathrm{age})=10.15$ (B08). Right: comparison between theoretical evolutionary isochrones $\mathrm{Z}=0.002, \mathrm{Y}=0.26, \log (\mathrm{age})=10.15$ (B08) (black) and the TERAMO isochrones $\mathrm{Z}=0.004, \mathrm{Y}=0.251$, age=14 Gyr (red) and $\mathrm{Z}=0.002, \mathrm{Y}=0.248$, age $=14$ (green).

$\log ($ age $)=10.10 ;$ (4) $\mathrm{Z}=0.002, \mathrm{Y}=0.30, \log ($ age $)=10.10$. There is no isochrone $\mathrm{Z}=0.002, \mathrm{Y}=$ $0.30, \log ($ age $)=10.15$ in the B08 model set. Comparison of these isochrones with the CMD of NGC 6652 is depicted in Figures A.6 and A.7 It can be seen in Fig. A.6 that isochrones (1) and (2) describe all the stellar evolutionary stages reasonably well, except for the aforementioned blue stars on the continuation of the HB. The synthetic Balmer lines appear to be narrow and shallow in comparison with the observed ones (see Fig. A.4). The same situation occurs in case we choose the isochrone (3) (see Figures A.5 (bottom) and A.7 (left)). If we choose isochrone (4), the synthetic Balmer lines demonstrate wider wings and deeper cores than the observed ones (see Figures A.5 (top) and A.7 (right)).

In the following we will address the question of why the TERAMO isochrones can not describe the spectrum as well. Fig. 2 (right panel) illustrates the comparison between three isochrones in the " $T_{\text {eff }}$ - luminosity" plane. It can be ascertained that slopes of the theoretical red giant branches are slightly different in case we choose for comparison the isochrones $\mathrm{Z}=0.002, \mathrm{Y}=0.26, \log (\operatorname{age})=10.15$ (B08) and $\mathrm{Z}=0.002, \mathrm{Y}=0.248$, age $=14 \mathrm{Gyr}(\mathrm{TERAMO})$. The ranges of $\mathrm{T}_{\mathrm{eff}}$ and $\operatorname{lgL}$ for HBs are also different. At the same time, $\mathrm{T}_{\text {eff }}$ and $\mathrm{LgL}$ data of other evolutionary stages agree well. If we choose for comparison the isochrones $\mathrm{Z}=0.002, \mathrm{Y}=0.26, \log ($ age $)=10.15(\mathrm{~B} 08)$ and $\mathrm{Z}=0.004, \mathrm{Y}=0.251$, age $=14$ Gyr (TERAMO), the shapes and slopes of all evolutionary sequences, except HB, look parallel. However, the second isochrone is noticeably shifted to the lower $\mathrm{T}_{\text {eff }}$. Additionally, the HB is cooler and less luminous in comparison with the isochrone by B08 .

\subsection{Abundances of chemical elements}

Most spectroscopic lines are blended at the resolution of $\lambda / \delta(\lambda) \sim 1600$. To determine chemical abundances, we selected dominant features in the spectra mostly sensitive to the abundances of the specific spectroscopic elements. The random errors of chemical abundances determined using our method and high 
Table 1: Estimated abundances $[\mathrm{X} / \mathrm{Fe}]$ in dex and abundance errors. Abundances from Conroy et al. 2018 (C18) are provided for comparison.

\begin{tabular}{lccccccccc}
\hline Ref./Elem. & $\mathrm{C}$ & $\mathrm{N}$ & $\mathrm{O}$ & $\mathrm{Na}$ & $\mathrm{Mg}$ & $\mathrm{Ca}$ & $\mathrm{Ti}$ & $\mathrm{Cr}$ \\
\hline ours & $0.20 \pm 0.14$ & $0.30 \pm 0.25$ & $0.3 \pm 0.25$ & $0.45 \pm 0.20$ & $0.40 \pm 0.10$ & $0.30 \pm 0.10$ & $-0.10 \pm 0.15$ & $-0.35 \pm 0.2$ & $-0.35 \pm 0.23$ \\
$\mathrm{C} 18$ & $0.02 \pm 0.02$ & $0.23 \pm 0.05$ & - & - & $0.45 \pm 0.02$ & $0.25 \pm 0.03$ & $0.25 \pm 0.03$ & - & - \\
\hline
\end{tabular}

sive absorption lines dominating the spectrum. These elements are $\mathrm{Fe}, \mathrm{C}, \mathrm{Mg}$ and $\mathrm{Ca}$. Comparison of our measurements for 20 Galactic GCs with the literature data obtained in high-resolution and IL spectroscopic studies indicates that the standard deviations of the differences between our and literature estimates of $[\mathrm{Fe} / \mathrm{H}],[\mathrm{Ca} / \mathrm{Fe}]$ and $[\mathrm{Mg} / \mathrm{Fe}]$ are $\sim 0.15 \mathrm{dex}$ (Sharina \& Shimansky 2019). The systematic shifts between our and literature data are $-0.06 \div-0.07$ dex for $[\mathrm{Ca} / \mathrm{Fe}]$ and $[\mathrm{Mg} / \mathrm{Fe}]$. The systematic shift between our and literature $[\mathrm{Fe} / \mathrm{H}]$ data is $\sim-0.2$ dex. We suppose that the reason is high microturbulence velocities $\xi_{\text {turb }}$ used by us (please, see Sec. 3.1 in Sharina et al. (2017) for a detailed description of the problem). The systematic difference of $0.37 \pm 0.17$ dex exists between our $[\mathrm{C} / \mathrm{Fe}]$ and literature $[\mathrm{C} / \mathrm{Fe}]$ values determined considering high resolution spectra (Sharina \& Shimansky 2019 and references therein). We interpret this result as the effect of a change in the chemical composition of the atmospheres of stars in the process of their evolution (Kraft 1994), because this difference vanishes while comparing our [C/Fe] estimates with the corresponding literature data from IL spectroscopy (Sharina \& Shimansky 2019 and references therein). The random errors of chemical abundances determined applying our method are larger for the elements with weak and blended lines $(\sigma \sim 0.15 \div 0.25 \mathrm{dex})$ : Ti, $\mathrm{Cr}, \mathrm{Mn}$ and $\mathrm{Na}$. The typical errors for the elements $\mathrm{N}$ and $\mathrm{O}$ are $\sigma \geq 0.3$ dex. These elements have spectroscopic features too weak to be detected in the studied spectral range. However, they influence the molecular and ionization equilibrium of other elements. Part of the $\mathrm{C}$ atoms forms the $\mathrm{CO}$ molecule if the $\mathrm{O}$ abundance is enhanced. This reduces the intensity of the molecular bands $\mathrm{CN}$ and $\mathrm{CH}$. Therefore, testing the equilibrium between different observed and theoretical intensities of these bands allows one to estimate abundances of all CNO group elements (Sharina et al. 2013, Sharina et al. 2017).

Table 1 shows the result of elemental abundances determination applying pixel-by-pixel fitting of the observed spectrum by the synthetic one. The determined metallicity is $[\mathrm{Fe} / \mathrm{H}]=-1.2$ dex with $\sigma \pm 0.1$ dex (random error corresponding to the internal accuracy of our method). Fig. A.3 features the continuum normalized IL spectrum of NGC 6652 (Schiavon et al. 2005) in a wide spectral range in comparison with the synthetic IL spectrum computed using the determined abundances (Table 1), the Ch05 MF and the isochrone $\mathrm{Z}=0.002, \mathrm{Y}=0.26, \log (\mathrm{age})=10.15(\mathrm{~B} 08)$, and smoothed to the resolution of the observed spectrum. Fig. A.8 depicts the continuum normalized spectrum of NGC 6652 (Schiavon et al. 2005) (black) in comparison with the synthetic IL spectrum (magenta) computed utilizing the isochrone $\mathrm{Z}=0.002, \mathrm{Y}=0.26, \log ($ age $)=10.15$ (B08), $[\mathrm{Fe} / \mathrm{H}]=-0.93$ dex, elemental abundances listed in Table 1 and the MF by Ch05.

\section{DISCUSSION}

As was mentioned in the introduction, the origin of NGC 6652 is a matter of debate. According to the 
(Bica et al. 2016, Massari et al. 2019). The origin of the bulge itself is not completely understood (Barbuy et al. 2018 and references therein). Stellar populations with chemical and kinematic properties from different Galactic subsystems are mixed there. The metallicity of NGC 6652 corresponds well to the peak of $[\mathrm{Fe} / \mathrm{H}]$ values for the majority of GCs in the bulge: $-1.3 \leq[\mathrm{Fe} / \mathrm{H}] \leq-0.8$, a metallicity peak similar to that of RR Lyrae stars. Without any doubt, this GC is one of the oldest in our Galaxy.

Prototypical bulge stars and GCs are enhanced in $\alpha$-process elements: $\mathrm{O}, \mathrm{Mg}, \mathrm{Ci}$, and $\mathrm{Ca}$ (Barbuy et al. 2018). The abundances of $\mathrm{Mg}$ and Ca confidently determined by us and by Conroy et al. (2018) correspond well to the values typical for bulge stellar populations with $[\mathrm{Fe} / \mathrm{H}] \sim-1 \mathrm{dex}$.

Sodium is among odd- $\mathrm{Z}$ elements produced together with $\mathrm{Mg}$ in massive stars. Its abundance is high in bulge stars (Barbuy et al. 2018). Our estimated abundance of $\mathrm{Na}$ is also high.

Manganese and chromium are Fe-peak elements. Low $[\mathrm{Mn} / \mathrm{Fe}]$ and $[\mathrm{Cr} / \mathrm{Fe}]$ values obtained by us are in line with the measurement for bulge stars (Barbuy et al. 2018).

Titanium is an iron peak element, but its enhancement depending on metallicity looks similar to that of $\alpha$-process elements (e.g. Barbuy et al. 2018). Our [Ti/Fe] value is too low in comparison to the value determined by Conroy et al. (2018). This element does not have intensive lines in the studied spectral range. Probably, our data is not accurate. However, let us note that there are other Galactic GCs in the bulge containing stars with reasonably low [Ti/Fe]: NGC 6522, NGC 6528, NGC 6553, NGC 6558 (Table 3 in Bica et al. 2016). Some of this objects reveal moderate $\alpha$-element enhancement. Galactic field RR Lyrae stars with $[\mathrm{Fe} / \mathrm{H}] \geq-1.0$ dex have unusually low [Ti/Fe] (Marsakov et al. 2019 and references therein). Low $[\mathrm{Ti} / \mathrm{Fe}]$ values are characteristic of stellar populations in dwarf galaxies (e.g. Carlin et al. 2018 and references therein). Future studies of Ti stellar abundances in NGC 6652 will definitely help to improve the data.

\section{CONCLUSIONS}

We use IL spectrum of the Galactic GC NGC 6652 (Schiavon et al. 2005) and our method (see e.g. Sharina \& Shimansky 2019, Sharina et al. 2018, Sharina et al. 2017) to derive the age $\sim 13.6 \pm 1 \mathrm{Gyr}, \mathrm{Y}=0.26 \pm 0.2$, $[\mathrm{Fe} / \mathrm{H}]=-1.2 \pm 0.1$ dex and abundances of chemical elements: $\mathrm{C}, \mathrm{N}, \mathrm{O}, \mathrm{Na}, \mathrm{Mg}, \mathrm{Ca}, \mathrm{Ti}, \mathrm{C}$ and $\mathrm{Mn}$. We conclude that the Ch05 MF and the isochrone $\mathrm{Z}=0.002, \mathrm{Y}=0.26, \log (\mathrm{age})=10.15$ (B08) allow us to reproduce well the shapes and intensities of the Balmer absorption lines in the observed spectrum. The determined metallicity is $0.2-0.3$ dex lower than the literature value (Zinn \& West 1984). This systematic deviation is specific for our analysis (Sharina \& Shimansky 2019). We argue that several hot HB stars contribute to the spectrum of NGC 6652 (Schiavon et al. 2005). The derived elemental abundances agree with the corresponding abundances of bulge stars and GCs (Bica et al. 2016, Barbuy et al. 2018). The only exclusion is $[\mathrm{Ti} / \mathrm{Fe}]$. The lines of this element are weak and blended at the examined spectral range and resolution. Future high-resolution spectroscopic studies will definitely help to establish the chemical composition of stars in NGC 6652 and the origin of this GC.

Acknowledgements We thank an anonymous referee for the report which helped to improve the paper. This work is supported by the RFBR grant No. 18-02-00167 a. 


\section{Appendix A: INTEGRATED-LIGHT SYNTHETIC SPECTRA COMPUTED USING DIFFERENT ISOCHRONES AND DIFFERENT MASS FUNCTIONS}

In this section, we illustrate the comparison between synthetic spectra computed using our method (see Sharina \& Shimansky 2019, Sharina et al. 2018, Sharina et al. 2017 and references therein), elemental abundances listed in Table1, and different isochrones and the comparison between IL spectrum of NGC 6652 (Schiavon et al. 2005), and the synthetic spectra computed applying different MFs: the Ch05 (equation 2 in that paper) and the Salpeter (1955) MFs.

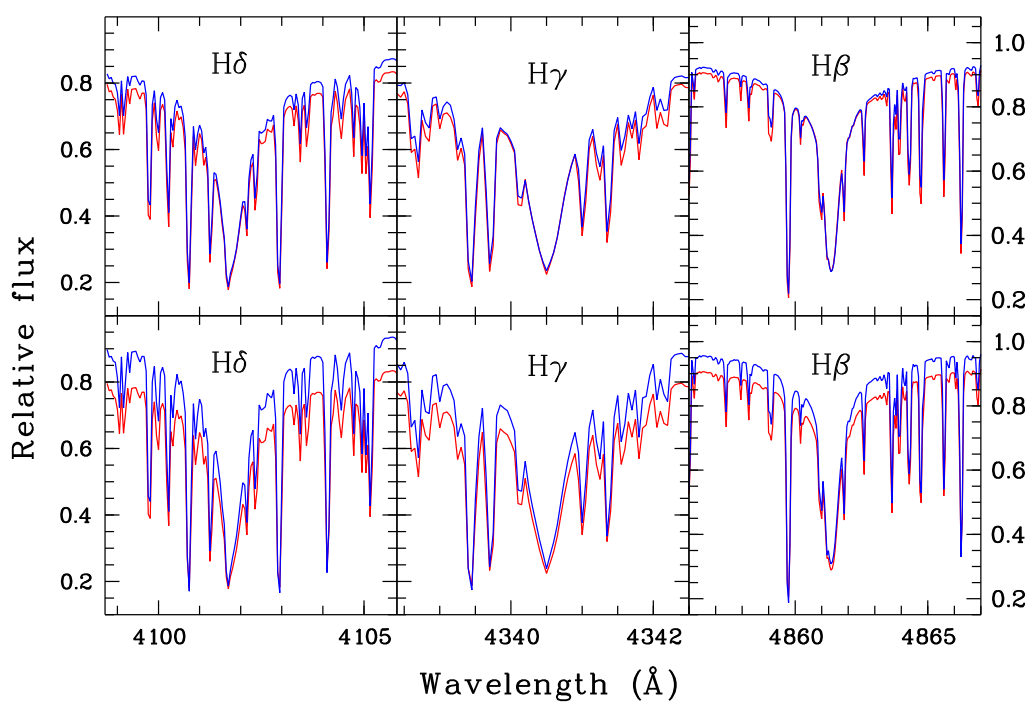

Fig. A.1: Comparison of three Balmer line profiles in the synthetic high-resolution spectra computed using the MF by $\mathrm{Ch} 05$, the same elemental abundances and different isochrones: the isochrone by B08 $\mathrm{Z}=0.002, \mathrm{Y}=0.26, \log ($ age $)=10.15$ displayed in red in the top and bottom panels and the isochrones by the TERAMO group (Pietrinferni et al. 2013 and references therein): $\mathrm{Z}=0.004, \mathrm{Y}=0.251$, age $=14 \mathrm{Gyr}$ and $\mathrm{Z}=0.002, \mathrm{Y}=0.248$, age $=14 \mathrm{Gyr}$ depicted in blue in the bottom and top panels, respectively.

Fig. A.1 illustrates the comparison between the original non-smoothed theoretical IL spectra computed using different isochrones: the isochrone by $\mathrm{B} 08 \mathrm{Z}=0.002, \mathrm{Y}=0.26, \log (\mathrm{age})=10.15$ signified in red in the top and bottom panels, the isochrones by the TERAMO group (Pietrinferni et al. 2013 and references therein) $\mathrm{Z}=0.004, \mathrm{Y}=0.251$, age $=14 \mathrm{Gyr}$ and $\mathrm{Z}=0.002, \mathrm{Y}=0.248$, age $=14 \mathrm{Gyr}$ depicted in blue in the bottom and top panels, respectively.

Fig. A.2 shows a comparison between the observed continuum normalized spectrum of NGC6652 and the synthetic IL spectra computed using the same isochrone and elemental abundances but different MFs. The synthetic spectra were smoothed to the resolution of the observed spectrum. It can be seen that the comparison is good in both cases and that there are no significant differences between the two synthetic IL spectra.

Fig. A.3 features the continuum normalized IL spectrum of NGC 6652 (Schiavon et al. 2005) in comparison with the synthetic IL spectrum computed utilizing the elemental abundances listed in Table1, the Ch05 MF and the isochrone $\mathrm{Z}=0.002, \mathrm{Y}=0.26, \log (\mathrm{age})=10.15$ (B08) and smoothed to the resolution of the 


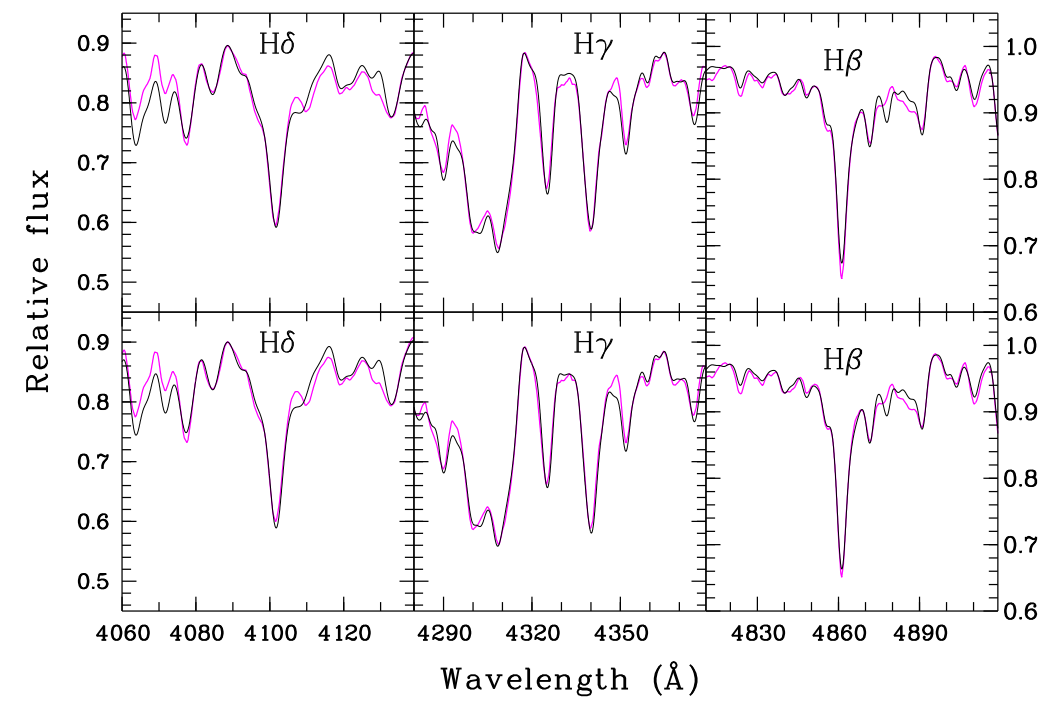

Fig. A.2: Comparison of three Balmer lines in the continuum normalized spectrum of NGC 6652 (Schiavon et al. 2005) (magenta) and the synthetic IL spectra (black) computed using the same isochrone $\mathrm{Z}=0.002, \mathrm{Y}=0.26, \log ($ age $)=10.15$ (B08) and elemental abundances but different MFs: the MF by Ch05 (bottom) and the MF by Salpeter (1955) (top).

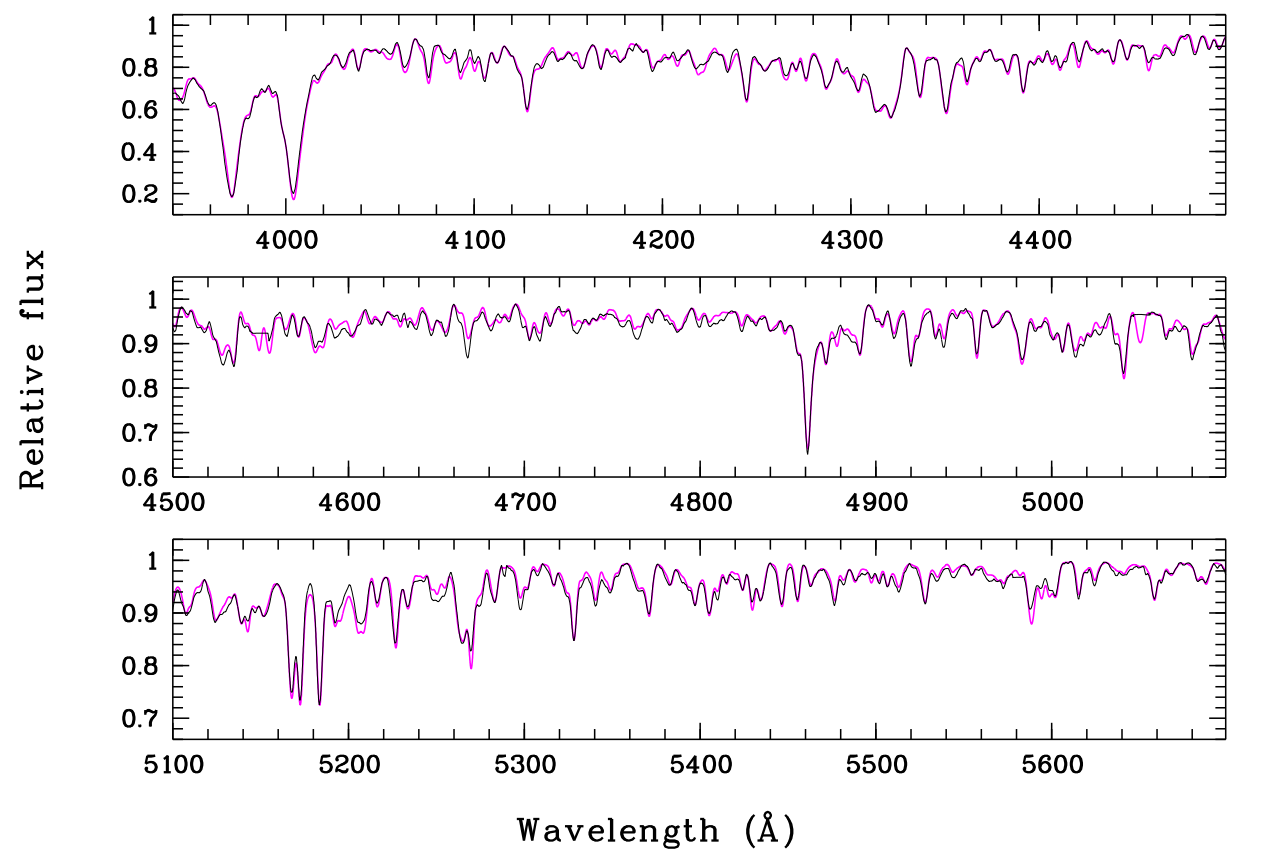

Fig. A.3: Comparison of the continuum normalized spectrum of NGC 6652 (Schiavon et al. 2005) (black) and the synthetic IL spectrum (magenta) computed using the isochrone $\mathrm{Z}=0.002, \mathrm{Y}=0.26, \log (\mathrm{age})=10.15$ (B08), the elemental abundances listed in Table 1 and the MF by Ch05.

the spectra by replacing each pixel value by the maximum of all points in the window $2 \times \mathrm{FWHM} * 10+1$, where FWHM is the average full width at half maximum for spectroscopic lines in the observed spectrum. Then, we implemented the running mean with the radius of $10 \times \mathrm{FWHM}$ to the filtered spectrum.

Figures A.4 and A.5 show the synthetic IL spectra computed in similar way as was described in the previous paragraph, but using the isochrones: $\mathrm{Z}=0.002, \mathrm{Y}=0.23, \log ($ age $)=10.15$ (Fig. A.4, bottom); $\mathrm{Z}=0.002$, 


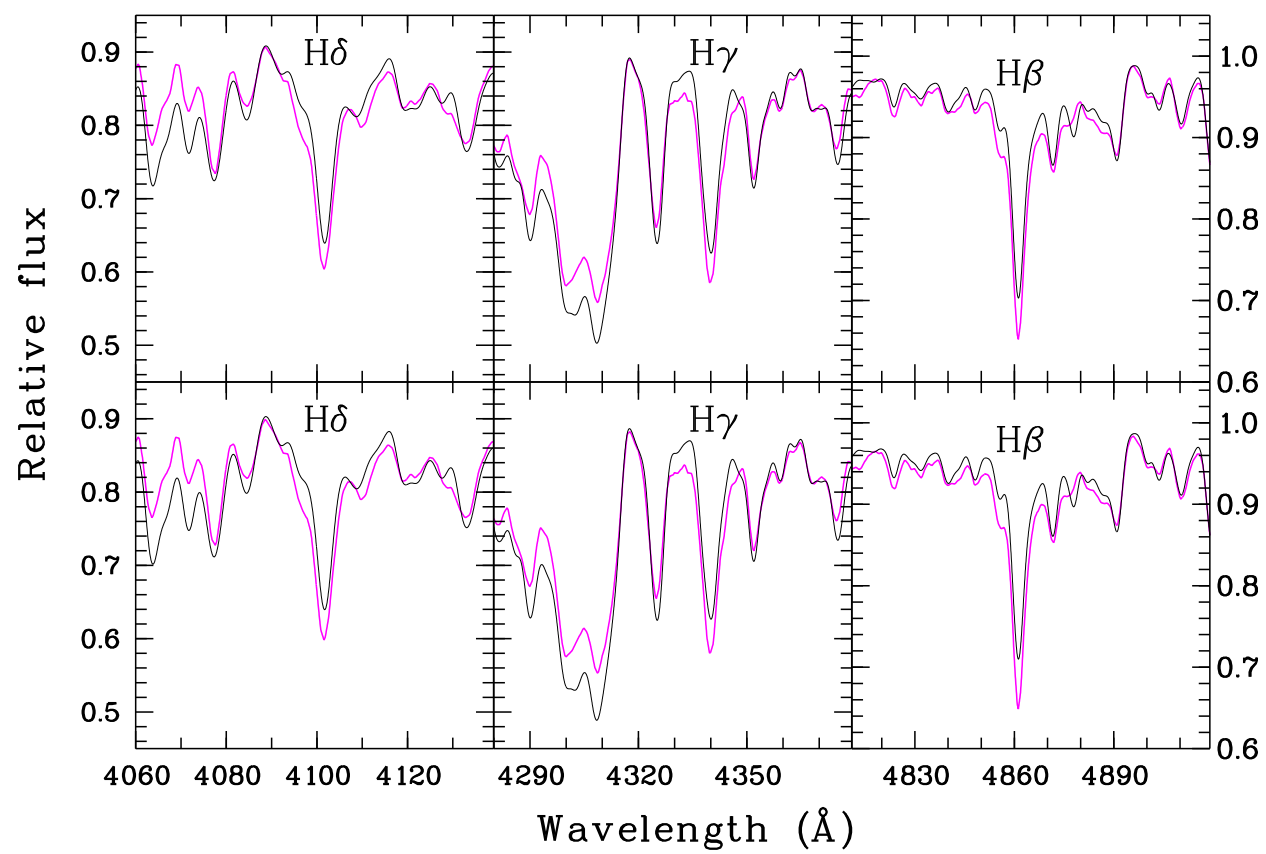

Fig. A.4: Comparison of three Balmer lines in the continuum normalized spectrum of NGC 6652 (Schiavon et al. 2005) (black) and the synthetic IL spectra (magenta) computed using the MF by Ch05, the same elemental abundances and different isochrones (B08): $\mathrm{Z}=0.002, \mathrm{Y}=0.23, \log (\mathrm{age})=10.15$ (bottom) and $\mathrm{Z}=0.002, \mathrm{Y}=0.23, \log ($ age $)=10.10$ (top).

$\mathrm{Y}=0.30, \log ($ age $)=10.10$ (Fig. A.5, top). The comparison between the CMD of NGC6652 (Sarajedini et al. 2007) and the aforementioned four theoretical evolutionary isochrones (B08) is depicted in Figures A.6 and A.7.

Fig. A.8 displays what happens if we apply $[\mathrm{Fe} / \mathrm{H}]=-0.93$ dex for the synthetic spectral calculation, the value obtained by Conroy et al. (2018). Other elemental abundances are listed in Table1.

\section{References}

Asplund M., Grevesse N., Sauval A. J., Scott P., Annual Review A\&A, 47, 481 (2009). 128

Balbinot E., Gieles M., 2018, MNRAS, 474, 2479129

Barbuy B., Chiappini C., Gerhard O., ARA\&A, 56, 223133

Bertelli G., Girardi L., Marigo P., Nasi E., 2008, A\&A, 484, 815 (B08) 129

Bica E., Ortolani S., Barbuy B., 2016, PASA, 33, 28 128, 133

Carlin J. L., Sheffield A. A., Cunha K., Smith V. V., 859, L10 133

Carretta E., \& Gratton R. G. 1997, A\&AS, 121, 95128

Castelli F. \& Kurucz R.L 2003, in: Piskunov N. et al. (eds.), Modeling of Stellar Atmospheres, Proc. IAU Symposium No.210 (Dordrecht: Kluwer), p. A20 129

Chaboyer, B., Sarajedini, A., Armandroff, T. E. 2000, AJ, 120, 3102128

Chabrier G., 2005, Astrophysics and Space Science Library, 327, 41 (Ch05) 129

Conroy C., Villaume A., van Dokkum P.G., Lind K., 2018, ApJ, 854, 139 128, 132, 133, 136

Kraft R.P., 1994, PASP, 106, 553132 


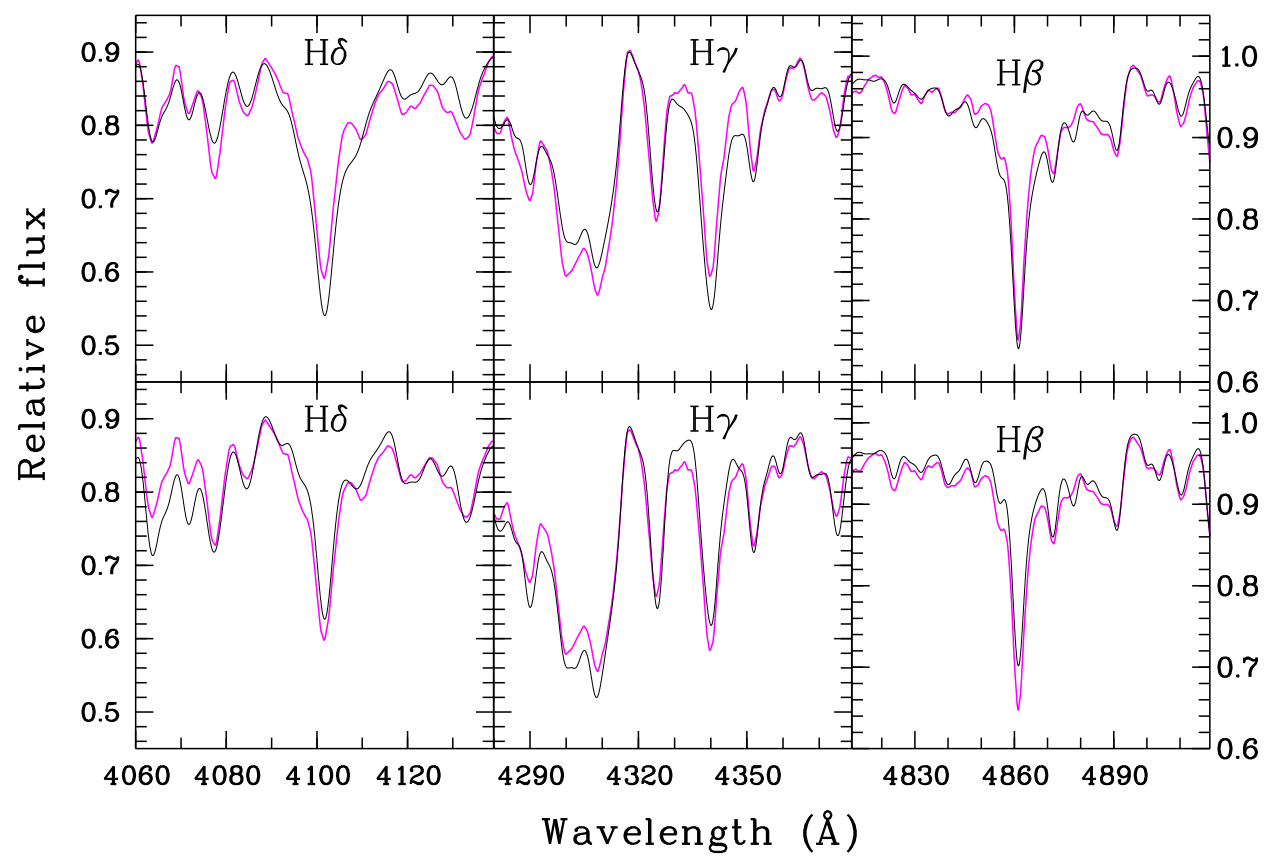

Fig. A.5: Comparison of three Balmer lines in the continuum normalized spectrum of NGC 6652 (Schiavon et al. 2005) (black) and the synthetic IL spectra (magenta) computed using the MF by Ch05, the same elemental abundances but different isochrones (B08): $\mathrm{Z}=0.002, \mathrm{Y}=0.26, \log ($ age $)=10.10$ (bottom) and $\mathrm{Z}=0.002, \mathrm{Y}=0.30, \log (\operatorname{age})=10.10$ (top).

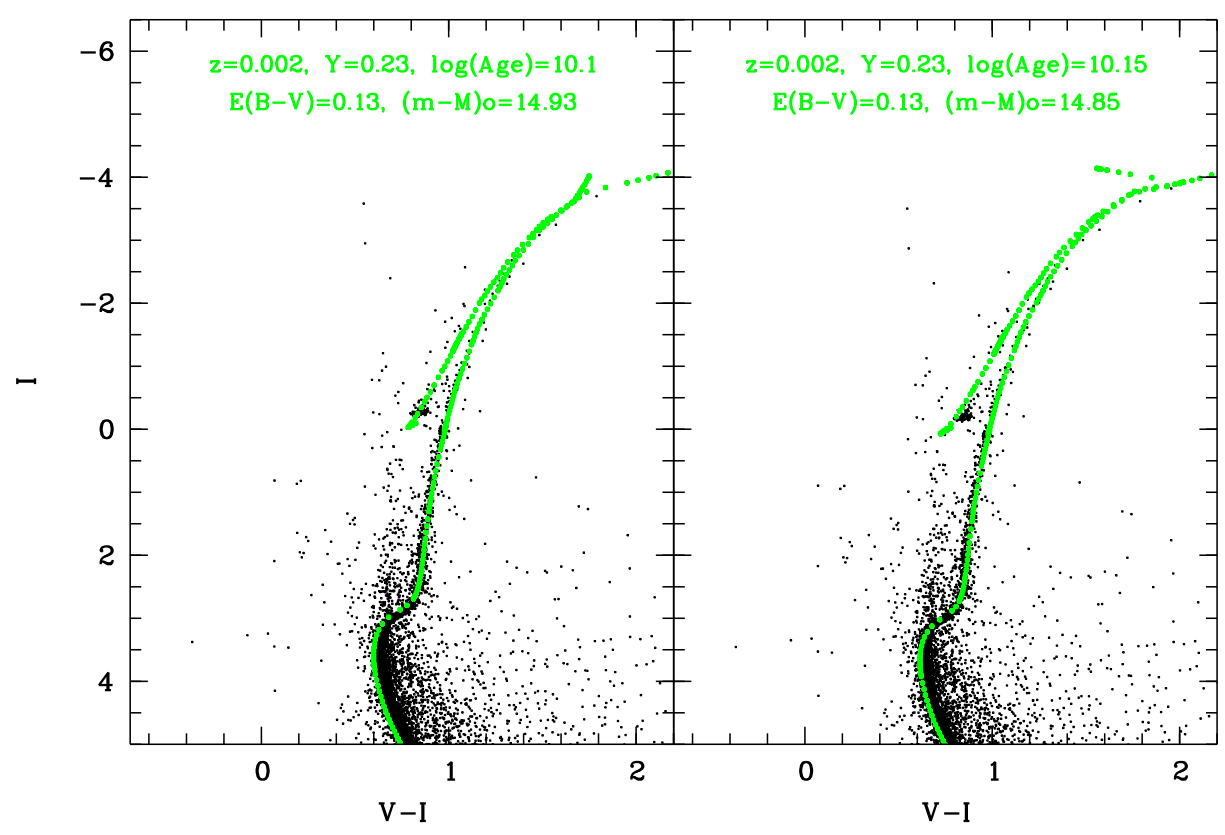

Fig. A.6: Comparison between the CMD of NGC6652 (Sarajedini et al. 2007) and the theoretical evolutionary isochrones (B08): $\mathrm{Z}=0.002, \mathrm{Y}=0.23, \log (\mathrm{age})=10.10$ (left) and $\mathrm{Z}=0.002, \mathrm{Y}=0.23, \log ($ age $)=10.15$ (right).

Massari D., Koppelman H. H., Helmi A., 2019, A\&A, 630, L4 133

Pietrinferni A., Cassisi S., Salaris M., Hidalgo S., 2013, A\&A, 558, 46 129, 134

Salpeter E., 1955, ApJ, 121, 161 130, 134, 135 


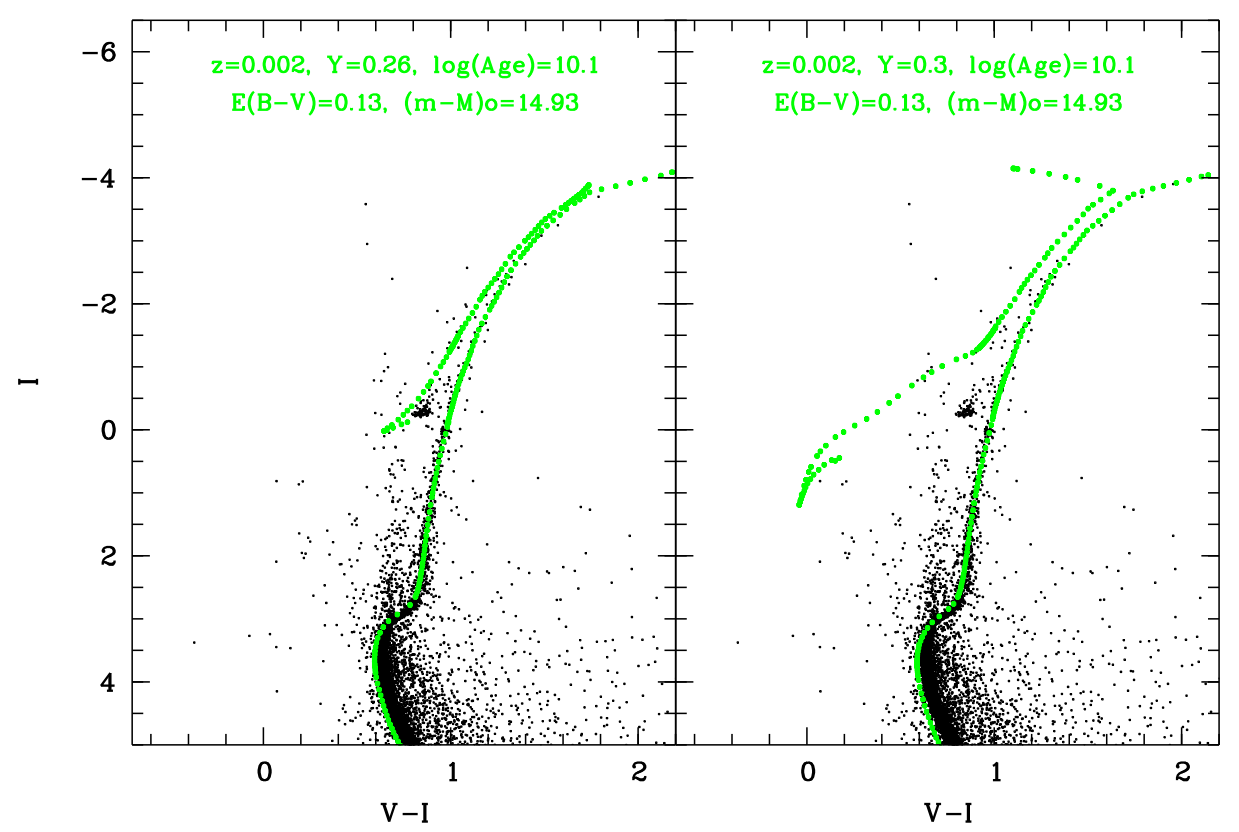

Fig. A.7: Comparison between the CMD of NGC6652 (Sarajedini et al. 2007) and the theoretical evolutionary isochrones (B08): $\mathrm{Z}=0.002, \mathrm{Y}=0.26, \log (\mathrm{age})=10.10$ (left) and $\mathrm{Z}=0.002, \mathrm{Y}=0.30, \log ($ age $)=10.10$ (right).

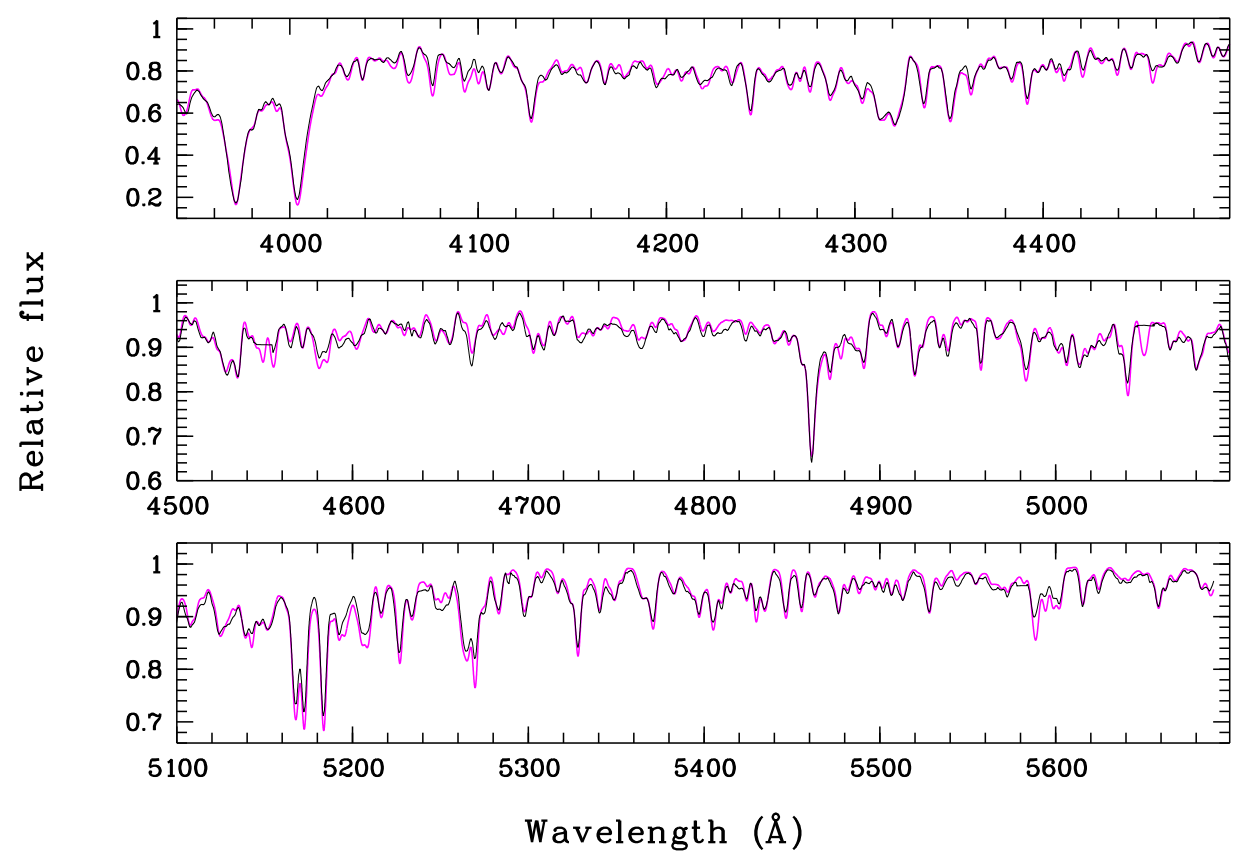

Fig. A.8: Comparison of the continuum normalized spectrum of NGC 6652 (Schiavon et al. 2005) (black) and the synthetic IL spectrum (magenta) computed using the isochrone $\mathrm{Z}=0.002, \mathrm{Y}=0.26, \log ($ age $)=10.15(\mathrm{~B} 08),[\mathrm{Fe} / \mathrm{H}]=$ -0.93 dex, elemental abundances listed in Table 1 and the MF by Ch05.

Schiavon R. P., Rose J. A., Courteau S., MacArthur L. A., 2005, ApJS, 160, 163 (Sch05) 128, 129, 130, $132,133,134,135,138$

Sharina M.E., Shimansky V.V., Davoust E., 2013, Astronomy Reports, 57, 410132 
Sharina M.E., Shimansky V.V., Khamidullina D.A., 2018, Astrophysical Bulletin, 73, 318 129, 133 , 134

Sharina M.E., Shimansky V.V., 2019, In: A. Bragaglia, M.B. Davies, A. Sills \& E. Vesperini, eds., Proc. IAU Symp. 351 "Star Clusters: From the Milky Way to the Early Universe", arXiv:1911.05976 129, 132, 133,134

Zinn R., \& West M. J., 1984, ApJS, 55, 45 128, 133 